\title{
ON A CERTAIN (MOD 2) IDENTITY AND A METHOD OF PROOF BY EXPANSION
}

\author{
RICHARD BLECKSMITH, JOHN BRILLHART, AND IRVING GERST \\ Beauty is often the child of imperfection
}

\begin{abstract}
We prove the congruence

$$
\prod_{\substack{n=1 \\ n \neq 7(\bmod 14)}}^{\infty}\left(1-x^{n}\right) \equiv \sum_{-\infty}^{\infty}\left(x^{n(3 n+2)}+x^{7 n(3 n+2)+2}\right)(\bmod 2)
$$

by first establishing a related equation, which reduces to the congruence modulo 2. The method of proof (called "expanding zero") is based on a formula of the authors for expanding the product of two triple products. A second proof of the result more fully explicates the various aspects of the method. A parity result for an associated partition function is also included.
\end{abstract}

\section{INTRODUCTION}

In two previous papers [1], [2] we proved a collection of identities which had initially been discovered as $(\bmod 2)$ congruences during a computer search, but which were later found to be equations over the integers when certain signs in them were changed. All these congruences led to parity theorems for the corresponding partition functions. (Cf. [3, Table 2].)

In this paper we prove another $(\bmod 2)$ identity, discovered during the abovementioned computer search, viz:

Theorem 1. There holds

$$
\prod_{\substack{n=1 \\ n \neq 7(\bmod 14)}}^{\infty}\left(1-x^{n}\right) \equiv \sum_{-\infty}^{\infty}\left(x^{n(3 n+2)}+x^{7 n(3 n+2)+2}\right) \quad(\bmod 2) .
$$

(Throughout this paper congruences will be understood to be $(\bmod 2)$.)

Unlike the $(\bmod 2)$ identities in [1] and [2], however, we found that this congruence had no equation over the integers of the same form standing behind

Received May 15, 1990.

1980 Mathematics Subject Classification (1985 Revision). Primary 05A19.

Key words and phrases. Jacobi triple product, quintuple product, mod 2 identity, expansion formula.

Research supported in part by NSF grant DMF8902258. 
it. (This conclusion was reached after an exhaustive search failed to find $\alpha_{n}$, $\beta_{n}$, and $\varepsilon_{n}$ equal to \pm 1 for which the equation

$$
\sum_{-\infty}^{\infty}\left(\alpha_{n} x^{n(3 n+2)}+\beta_{n} x^{7 n(3 n+2)+2}\right)=\prod_{\substack{n=1 \\ n \neq 7(\bmod 14)}}^{\infty}\left(1+\varepsilon_{n} x^{n}\right)
$$

holds. (Cf. [2, §6].) However, since the identities proved in [1] and [2] were of interest in themselves and easily implied the mod 2 results, we take as our first task here the discovery of an equation that is equivalent to (1) modulo 2 . This is accomplished in $\S 2$, where the desired equation (9) is derived. Also presented in $\S 2$ is the partition parity result associated with (1) (Theorem 3 ). The rest of the paper is then devoted to proving (9). (Actually we present two proofs and a discussion of the methods employed.)

The first proof is given in two parts in $\S 3$. The first part contains a transformation of (9) into equation (16) by means of the expansion formula developed in [2] and first used there. The second part contains the transformation of equation (16) into equation (17), which in turn is changed into the final equation (20). Equation (20) is then proved using a device we call an "expansion of zero". Since all of these steps are reversible, we have that $(9) \leftrightarrow(16) \leftrightarrow(17) \leftrightarrow(20)$.

In $\S 4$ we derive a further expansion formula and discuss other expansions of zero. The second proof of $(9)$ is then presented in $\S 5$. Although this proof employs the same ideas as the proof of (20), it establishes (9) without the use of the quintuple product. Since the second proof is more elaborate, we follow it in $\S 6$ with a discussion of the ideas and analytic methods which were used in constructing that proof, such as the two algorithms, Forward and Backward, that allow us to discover which expansions are available to use. The second proof was primarily presented here to show that a proof of equation (9) (and similar identities) could be found in a somewhat systematic way and that the large number of terms which were generated by the expansions in the proof could readily be managed by using a computer.

\section{THE DERIVATION OF AN EQUATION THAT IMPLIES CONGRUENCE (1)}

We begin by recalling some material from [2, pp. 302-303]. Let $r_{1}, \ldots, r_{t}$ be distinct residues modulo $m$ and let $S=\left\{n \in Z^{+}: n \equiv r_{1}, \ldots, r_{t}(\bmod m)\right\}$. Then $\left(r_{1}, \ldots, r_{t}\right)_{m}$ will denote the infinite product $\prod_{n \in S}\left(1-x^{n}\right)$ and $\left[r_{1}, \ldots, r_{t}\right]_{m}$ will denote $\prod_{n \in S}\left(1+x^{n}\right)$.

For $\delta, \varepsilon \in\{0,1\}$, we define the four one-variable $T$-functions by the formula

$$
\begin{aligned}
T_{2 \delta+\varepsilon}(k, l) & \stackrel{\text { def }}{=} \sum_{-\infty}^{\infty}(-1)^{\delta \frac{\eta n+1)}{2}-n \varepsilon} x^{k n^{2}+l n} \\
= & \prod_{n=1}^{\infty}\left[1-(-1)^{n \delta} x^{2 k n}\right]\left[1+(-1)^{n \delta+\varepsilon} x^{2 k n-k+l}\right] \\
& \cdot\left[1+(-1)^{(n+1) \delta+\varepsilon} x^{2 k n-k-l}\right],
\end{aligned}
$$


the Jacobi triple products being expressed concisely in the above notation as

$$
\begin{aligned}
& T_{0}(k, l)=(0)_{2 k}[ \pm(k-l)]_{2 k}, \\
& T_{1}(k, l)=(0, \pm(k-l))_{2 k}, \\
& T_{2}(k, l)=(0, \pm(k+l))_{4 k}[ \pm(k-l), 2 k]_{4 k}, \\
& T_{3}(k, l)=(0, \pm(k-l))_{4 k}[ \pm(k+l), 2 k]_{4 k} .
\end{aligned}
$$

(For simplicity we usually write $T$ for $T_{0}$.)

We also have the Gauss formula [2, (13)]

$$
\frac{1}{2} T\left(\frac{n}{2}, \frac{n}{2}\right)=\frac{(0)_{2 n}}{(n)_{2 n}}=T(2 n, n) .
$$

To begin the derivation, we use (2) in (1), obtaining

$$
\begin{aligned}
\prod_{\substack{n=1 \\
n \equiv 7(\bmod 14)}}^{\infty}\left(1-x^{n}\right) & \equiv \sum_{-\infty}^{\infty}(-1)^{n} x^{3 n^{2}+2 n}+x^{2} \sum_{-\infty}^{\infty}(-1)^{n} x^{21 n^{2}+14 n} \\
& =T_{1}(3,2)+x^{2} T_{1}(21,14) .
\end{aligned}
$$

Writing all the terms in product form gives

$$
(0, \pm 1)_{6}+x^{2}(0, \pm 7)_{42} \equiv \frac{(0)_{1}}{(7)_{14}} \text {. }
$$

Next, by [3, Ex. 4] and [2, (18)], we have

$$
( \pm 1)_{6} \equiv \frac{1}{( \pm 1)_{3}(0)_{6}(6)_{12}} \equiv \frac{1}{( \pm 1)_{3}}
$$

and its companion (by $x \rightarrow x^{7}$ )

$$
( \pm 7)_{42} \equiv \frac{1}{( \pm 7)_{21}}
$$

Thus, (5) becomes

$$
\begin{aligned}
\frac{(0)_{6}}{( \pm 1)_{3}}+x^{2} \frac{(0)_{42}}{( \pm 7)_{21}} & \equiv \frac{(0)_{1}}{(7)_{14}}=\frac{(0)_{14}(0)_{1}^{2}}{(0,7)_{14}(0)_{1}} \\
& \equiv \frac{(0)_{14}(0)_{2}}{(0)_{7}(0)_{1}}=\frac{(0)_{14}(0)_{2}}{(0)_{21}( \pm 7)_{21}(0)_{3}( \pm 1)_{3}}
\end{aligned}
$$

Clearing fractions gives

$$
(0)_{21}( \pm 7)_{21}(0)_{3}(0)_{6}+x^{2}(0)_{42}(0)_{21}(0)_{3}( \pm 1)_{3} \equiv(0)_{14}(0)_{2} \text {. }
$$

By $[2,(18)]$, we have $(0)_{3} \equiv \frac{1}{(3)_{6}}$, and so $(0)_{21} \equiv \frac{1}{(21)_{42}}$. Thus, (6) becomes

$$
(0)_{21}( \pm 7)_{21} \frac{(0)_{6}}{(3)_{6}}+x^{2} \frac{(0)_{42}}{(21)_{42}}(0)_{3}( \pm 1)_{3} \equiv(0)_{14}(0)_{2} \text {. }
$$


As this congruence stands, it is not an equation, which can readily be determined by comparing the power series expansions of the terms on the two sides. But one finds, after numerous trial sign changes, that the following is an equation:

$$
(0)_{21}[ \pm 7]_{21} \frac{(0)_{6}}{(3)_{6}}-x^{2} \frac{(0)_{42}}{(21)_{42}}(0)_{3}[ \pm 1]_{3}=(0, \pm 14)_{42}(0, \pm 2)_{6} \text {. }
$$

(Working with power series, one finds that the two sides of (8) agree up to degree 1000 , which implies heuristically that (8) is an equation.) Using (3) and (4), we can express (8) in terms of $T$-functions, thereby obtaining an equation of the kind we wished to derive:

Theorem 2. We have

$$
T\left(\frac{21}{2}, \frac{7}{2}\right) T\left(\frac{3}{2}, \frac{3}{2}\right)-x^{2} T\left(\frac{21}{2}, \frac{21}{2}\right) T\left(\frac{3}{2}, \frac{1}{2}\right)=2 T_{1}(21,7) T_{1}(3,1) .
$$

Two proofs of Theorem 2 are given respectively in $\S \S 3$ and 5. It is also clear from the way that (9) was derived from (1) that congruence (1) will be true if equation (9) is shown to be true.

Before proceeding to the proof of Theorem 2, we will give two consequences of Theorem 1. Recall the definition of the $Q$-function and the Quintuple Product formula in our notation [2, p. 304], viz.

$$
\begin{aligned}
Q(m, k) & \stackrel{\text { def }}{=} \sum_{-\infty}^{\infty} x^{\frac{m\left(3 n^{2}+n\right)}{2}}\left(x^{-3 k n}-x^{3 k n+k}\right) \\
& =T\left(\frac{3 m}{2}, \frac{m}{2}-3 k\right)-x^{k} T\left(\frac{3 m}{2}, \frac{m}{2}+3 k\right) \\
& =(0, \pm k, \pm(m-2 k), \pm(m-k), m)_{2 m} .
\end{aligned}
$$

Corollary. Let $S=\left\{n \in Z^{+}: n \not \equiv 21(\bmod 42)\right\}$. Then

$$
x \prod_{n \in S}\left(1-x^{n}\right) \equiv \sum_{-\infty}^{\infty}\left(x^{(3 n+1)^{2}}+x^{7(3 n+1)^{2}}\right) \equiv Q(6,1)+Q(42,7) .
$$

Proof. Replacing $x$ by $x^{3}$ in (1) and then multiplying by $x$ gives the first congruence. Then (10) gives

$$
Q(6,1)=\sum_{-\infty}^{\infty} x^{9 n^{2}}-\sum_{-\infty}^{\infty} x^{(3 n+1)^{2}} \equiv 1+\sum_{-\infty}^{\infty} x^{(3 n+1)^{2}},
$$

from which the second congruence follows.

Theorem 3. Let $S=\left\{n \in Z^{+}: n \equiv \pm(1,3,5,9,11,13), 14(\bmod 28)\right\}$ and let $p(S ; k)$ denote the number of partitions of $k$ whose parts lie in $S$. Then $p(S ; k)$ is odd if and only if $k=3 n^{2} \pm n$ or $21 n^{2} \pm 7 n+2, n \geq 0$. 
Proof. Using the algorithm in [3, §4] for finding the Euler reciprocal (mod 2), we discover that

$$
\begin{gathered}
\frac{1}{(0, \pm 1, \pm 2, \pm 3, \pm 4, \pm 5, \pm 6)_{14}} \equiv \frac{(0, \pm 2, \pm 4, \pm 6)_{14}( \pm 1, \pm 3, \pm 5,7)_{14}^{2}}{(0, \pm 1, \pm 2, \pm 3, \pm 4, \pm 5, \pm 6)_{14}} \\
=( \pm 1, \pm 3, \pm 5)_{14}(7)_{14}^{2} \equiv( \pm 1, \pm 3, \pm 5, \pm 9, \pm 11, \pm 13,14)_{28}(\bmod 2) .
\end{gathered}
$$

Thus, congruence (1) implies

$$
\begin{aligned}
1+\sum_{k=1}^{\infty} p(S ; k) x^{k} & =\prod_{n \in S} \frac{1}{1-x^{n}} \equiv \prod_{\substack{n=1 \\
n \neq 7(\bmod 14)}}^{\infty}\left(1-x^{n}\right) \\
& \equiv 1+\sum_{n=1}^{\infty}\left(x^{n(3 n \pm 1)}+x^{7 n(3 n \pm 1)+2}\right)
\end{aligned}
$$

from which the result follows.

\section{THE FIRST PROOF OF THEOREM 2}

We begin this section by observing that each term of (9) is the product of two $T$ 's-two terms with $T_{0} \cdot T_{0}$ and the third with $T_{1} \cdot T_{1}$. Because of the importance of an equation with this form, we introduce the following terminology.

Definition. A set of terms, or an equation, will be called a " $T^{2}$ set" or a " $T^{2}$ equation", respectively, if each of its nonzero terms has the form $a x^{\alpha} T_{\varepsilon_{1}} \cdot T_{\varepsilon_{2}}$, where $a$ is a constant and $\varepsilon_{1}, \varepsilon_{2} \in\{0,1,2,3\}$. (Our use of " $T$ " here is not short for " $T_{0}$ ".) Corresponding terminology will also be used for $Q^{2}$ sets and $Q^{2}$ equations.

In [2, p. 306] we developed the following formula for expanding the singlevariable product $T_{\varepsilon_{1}}\left(k_{1}, l_{1}\right) T_{\varepsilon_{2}}\left(k_{2}, l_{2}\right), \varepsilon_{1}, \varepsilon_{2} \in\{0,1\}$, into a finite sum of terms which form a $T^{2}$ set. We shall refer to this as "the expansion formula". It will be used extensively throughout the rest of the paper.

Expansion Formula. Let $a, b, m \in Z^{+}$and $\left(k_{1}, l_{1}\right),\left(k_{2}, l_{2}\right) \in\left\{\left(\frac{i}{2}, \frac{j}{2}\right)\right.$ : $\left.(i, j) \in Z^{+} \times Z, i \equiv j(\bmod 2)\right\}$ and assume $\varepsilon_{1}, \varepsilon_{2} \in\{0,1\}$. If the separability condition

$$
k_{1} b=k_{2} a(m-a b)
$$

is satisfied, then

$$
T_{\varepsilon_{1}}\left(k_{1}, l_{1}\right) T_{\varepsilon_{2}}\left(k_{2}, l_{2}\right)=\sum_{r \in R}(-1)^{\varepsilon_{2} r} x^{k_{2} r^{2}+l_{2} r} T_{\delta_{1}}\left(K_{1}, L_{1}(r)\right) T_{\delta_{2}}\left(K_{2}, L_{2}(r)\right),
$$


where

$$
\begin{array}{cc}
K_{1}=k_{1}+k_{2} a^{2}, & K_{2}=k_{2} m(m-a b), \\
L_{1}(r)=l_{1}-l_{2} a-2 k_{2} a r, & L_{2}(r)=\left(2 k_{2} r+l_{2}\right)(m-a b)+l_{1} b, \\
\delta_{1}=\left(\varepsilon_{1}+\varepsilon_{2} a\right) \bmod 2, & \delta_{2}=\left(\varepsilon_{1} b+\varepsilon_{2}(m-a b)\right) \bmod 2,
\end{array}
$$

and $R$ is a complete residue system $\bmod m$.

(In (12), the expression $x$ mod 2 denotes the remainder-0 or 1-upon dividing $x$ by 2.) We should point out that whenever this formula is used, all the terms in the expansion have the same $K_{1}, K_{2}$ pair and the same $\delta_{1}, \delta_{2}$ pair, since these quantities are not functions of $r$. These invariant numbers are central to the uses we will make of this formula in the proofs that follow. In particular, they show that a $T^{2}$ equation transforms into a $T^{2}$ equation. (Whenever we list the parameters $a, b, m$ in this expansion, we will write them together in the notation $[a, b, m]$.)

After using the expansion formula, which has $m$ terms, it is important to reduce the terms so they can be properly combined.

Definition. If $\varepsilon \in\{0,1\}$, then the function $T_{\varepsilon}(k, l)$ is said to be reduced, or in reduced form, if $0 \leq l \leq k$.

Because of the property (cf. $[2,(14)]$ )

$$
T_{\varepsilon}(k,-l)=T_{\varepsilon}(k, l), \quad \varepsilon \in\{0,1\},
$$

we can assume $l \geq 0$. (For $T_{2}$ and $T_{3}$ we have the formula $T_{2}(k,-l)=$ $T_{3}(k, l)$.) If $l>k$, we can reduce the value in the second argument by applying the following single-step reduction formula and (13)-over and over if necessary-until $T$ is in reduced form.

Reduction Formula. For $k, l \in Z^{+}, k<l$, and $\varepsilon \in\{0,1\}$,

$$
x^{r} T_{\varepsilon}(k, l)=(-1)^{\varepsilon} x^{r-(l-k)} T_{\varepsilon}(k, 2 k-l) .
$$

Proof. From (2) we have

$$
\begin{aligned}
x^{r} T_{\varepsilon}(k, l) & =x^{r} \sum_{-\infty}^{\infty}(-1)^{\varepsilon n} x^{k n^{2}+l n}=x^{r} \sum_{-\infty}^{\infty}(-1)^{\varepsilon(-n-1)} x^{k(-n-1)^{2}+l(-n-1)} \\
& =(-1)^{\varepsilon} x^{r-(l-k)} \sum_{-\infty}^{\infty}(-1)^{\varepsilon n} x^{k n^{2}+(2 k-l) n} \\
& =(-1)^{\varepsilon} x^{r-(l-k)} T_{\varepsilon}(k, 2 k-l) .
\end{aligned}
$$

This kind of reduction was used previously in [2, p. 309], where it was worked out separately in each case. (The same reduction formula holds for $T_{2}$ and $T_{3}$.) 
Lemma 1. If $\varepsilon \in\{0,1\}$, then

$$
T_{\varepsilon}(k, l)=T(4 k, 2 l)+(-1)^{\varepsilon} x^{k-l} T(4 k, 4 k-2 l) .
$$

Proof. This expansion comes from splitting the index values in the sum on the left into even and odd parts.

Also, from [2, (15)] we have

Lemma 2. We have $T_{1}\left(\frac{k}{2}, r \frac{k}{2}\right)=0$, where $k$ is a positive integer and $r$ is an odd integer.

We are now in a position to give the first proof of (9).

First Proof of Theorem 2. The proof falls naturally into two parts.

Part 1. Our purpose here is to use the expansion formula to transform the terms of equation (9) equivalently into equation (16), an equation that involves only the $Q$-function defined in (10).

We first transform the two terms on the left of (9) by the same expansion with the parameters $[1,1,8]$. (Note that $(11)$ is satisfied.) This gives two groups of eight $T \cdot T$ terms, so the left-hand side of (9) with the $T$ 's reduced becomes

$$
\begin{aligned}
& x^{16} T(12,10) T(84,70)+x^{9} T(12,11) T(84,49)+x^{3} T(12,8) T(84,28) \\
& +T(12,5) T(84,7)+T(12,2) T(84,14)+x^{3} T(12,1) T(84,35) \\
& +x^{9} T(12,4) T(84,56)+x^{18} T(12,7) T(84,77) \\
& -x^{2}\left[x^{12} T(12,2) T(84,70)+x^{5} T(12,5) T(84,49)\right. \\
& \quad+x T(12,8) T(84,28)+T(12,11) T(84,7) \\
& \quad+T(12,10) T(84,14)+x^{2} T(12,7) T(84,35) \\
& \left.+x^{7} T(12,4) T(84,56)+x^{15} T(12,1) T(84,77)\right] .
\end{aligned}
$$

Since the third and seventh terms in the first group respectively cancel the corresponding terms in the second group, we get the equation

$$
\begin{aligned}
T\left(\frac{21}{2},\right. & \left.\frac{7}{2}\right) T\left(\frac{3}{2}, \frac{3}{2}\right)-x^{2} T\left(\frac{21}{2}, \frac{21}{2}\right) T\left(\frac{3}{2}, \frac{1}{2}\right) \\
= & T(12,2) T(84,14)+T(12,5) T(84,7) \\
& +x^{3} T(12,1) T(84,35)+x^{9} T(12,11) T(84,49) \\
& +x^{16} T(12,10) T(84,70)+x^{18} T(12,7) T(84,77) \\
& -x^{2} T(12,10) T(84,14)-x^{2} T(12,11) T(84,7) \\
& -x^{4} T(12,7) T(84,35)-x^{7} T(12,5) T(84,49) \\
& -x^{14} T(12,2) T(84,70)-x^{17} T(12,1) T(84,77) .
\end{aligned}
$$


Rearranging and grouping the terms on the right of (15), we obtain

$$
\begin{aligned}
T\left(\frac{21}{2}, \frac{7}{2}\right) T\left(\frac{3}{2}, \frac{3}{2}\right)-x^{2} T\left(\frac{21}{2}, \frac{21}{2}\right) T\left(\frac{3}{2}, \frac{1}{2}\right) & \\
= & {\left[T(12,5) T(84,7)-x^{2} T(12,11) T(84,7)-x^{7} T(12,5) T(84,49)\right.} \\
& \left.+x^{9} T(12,11) T(84,49)\right] \\
+ & x^{3}[T(12,1) T(84,35)-x T(12,7) T(84,35) \\
& \left.\quad-x^{14} T(12,1) T(84,77)+x^{15} T(12,7) T(84,77)\right] \\
& +\left[T(12,2) T(84,14)-x^{2} T(12,10) T(84,14)\right. \\
& \left.\quad-x^{14} T(12,2) T(84,70)+x^{16} T(12,10) T(84,70)\right] \\
= & {\left[T(12,5)-x^{2} T(12,11)\right]\left[T(84,7)-x^{7} T(84,49)\right] } \\
& +x^{3}[T(12,1)-x T(12,7)]\left[T(84,35)-x^{14} T(84,77)\right] \\
& +\left[T(12,2)-x^{2} T(12,10)\right]\left[T(84,14)-x^{14} T(84,70)\right] \\
= & Q(8,3) Q(56,7)+x^{3} Q(8,1) Q(56,21)+T_{1}(3,1) T_{1}(21,7) .
\end{aligned}
$$

(Here, (10) was used on the first four brackets, and Lemma 1 on the last two.) But the $T_{1}$ 's in the third term are also $Q$ 's, since $T_{1}(3,1)=(0)_{2}=Q(8,2)$, and so $T_{1}(21,7)=(0)_{14}=Q(56,14)$ (by $\left.x \rightarrow x^{7}\right)$. If we now equate this expression to the left-hand side of (9) (which equals $2 Q(8,2) Q(56,14)$ ), we arrive at the remarkable $Q^{2}$ equation

$$
Q(8,3) Q(56,7)+x^{3} Q(8,1) Q(56,21)=Q(8,2) Q(56,14) .
$$

Part 2. Our next purpose is to transform (16) into (17) and then to prove (17) by using the expansion formula and an expansion of zero.

To begin, write the six $Q$ 's in (16) in their product form using (10), viz.

$$
\begin{aligned}
& Q(8,3)=(0)_{8}( \pm 2)_{16}( \pm 3)_{8}=( \pm 1)_{8}( \pm 3)_{8} \cdot(0)_{8}[ \pm 1]_{8}, \\
& Q(8,1)=(0)_{8}( \pm 6)_{16}( \pm 1)_{8}=( \pm 1)_{8}( \pm 3)_{8} \cdot(0)_{8}[ \pm 3]_{8}, \\
& Q(56,7)=( \pm 7)_{56}( \pm 21)_{56} \cdot(0)_{56}[ \pm 21]_{56}, \\
& Q(56,21)=( \pm 7)_{56}( \pm 21)_{56} \cdot(0)_{56}[ \pm 7]_{56}, \\
& Q(8,2)=(0)_{2} \text { and } Q(56,14)=(0)_{14} .
\end{aligned}
$$

Substituting these products into (16), dividing by $( \pm 1, \pm 3)_{8}( \pm 7, \pm 21)_{56}$, and using (3) gives

$$
T(4,3) T(28,7)+x^{3} T(4,1) T(28,21)=\frac{(0)_{2}(0)_{14}}{( \pm 1, \pm 3)_{8}( \pm 7, \pm 21)_{56}}=\frac{(0)_{2}(0)_{14}}{(1)_{2}(7)_{14}} \text {. }
$$

By (4), we have $\frac{(0)_{2}}{(1)_{2}}=\frac{1}{2} T\left(\frac{1}{2}, \frac{1}{2}\right)$ and $\frac{(0)_{14}}{(7)_{14}}=\frac{1}{2} T\left(\frac{7}{2}, \frac{7}{2}\right)$. Substituting these into the term on the far right, we obtain the new equation

$$
4 T(4,3) T(28,7)+4 x^{3} T(4,1) T(28,21)=T\left(\frac{1}{2}, \frac{1}{2}\right) T\left(\frac{7}{2}, \frac{7}{2}\right) .
$$


Note that the terms on the left of (17) have the $k$-pair $k_{1}=4, k_{2}=28$, while the term on the right has $k_{1}=\frac{1}{2}, k_{2}=\frac{7}{2}$. This difference suggests the following question: Can the right-hand side of (17) be expanded to produce terms with the $k$-pair $(4,28)$ or $(28,4)$ ? The answer is "yes"; for if we take $k_{1}=\frac{7}{2}, k_{2}=\frac{1}{2}$ with the parameters $[1,1,8]$, we find that

$$
T\left(\frac{7}{2}, \frac{7}{2}\right) T\left(\frac{1}{2}, \frac{1}{2}\right)=\sum_{r=-4}^{3} x^{\frac{1}{2}\left(r^{2}+r\right)} T(4,3-r) T(28,7 r+7),
$$

or after reducing,

$$
\begin{aligned}
T\left(\frac{7}{2}, \frac{7}{2}\right) T\left(\frac{1}{2}, \frac{1}{2}\right)= & 2 T(4,3) T(28,7)+2 x^{3} T(4,1) T(28,21) \\
& +2 x T(4,2) T(28,14)+x^{6} T(4,0) T(28,28) \\
& +T(4,4) T(28,0) .
\end{aligned}
$$

Equating the left-hand side of (17) to the right-hand side of (19) gives

$$
\begin{aligned}
2 T(4,3) T(28,7) & +2 x^{3} T(4,1) T(28,21)-2 x T(4,2) T(28,14) \\
& -x^{6} T(4,0) T(28,28)-T(4,4) T(28,0)=0
\end{aligned}
$$

Now observe that the terms on the left of (20) are the same as those on the right of (19) except for the minus signs. Also note that the negative terms occur in the sum in (18) at the odd values of $r$. Thus, (20) can be written as the alternating sum

$$
\sum_{r=-4}^{3}(-1)^{r} x^{\frac{1}{2}\left(r^{2}+r\right)} T(4,3-r) T(28,7 r+7)=0 .
$$

But this equation is valid since the left-hand side results from applying the expansion theorem to $T_{1}\left(\frac{7}{2}, \frac{7}{2}\right) T_{1}\left(\frac{1}{2}, \frac{1}{2}\right)$ with parameters [1,1,8], and the latter product is zero by Lemma 2 . This establishes equation (9).

Remark. In the rest of this paper the phrase "expansion of zero" will be used to designate a linear sum of $T^{2}$ terms, say $f(x)$, which arise by applying the expansion theorem to a single $T T$ term which is identically zero. Then the $T^{2}$ equation $f(x)=0$ holds. Thus, the left-hand side of (21) is an example of an expansion of zero.

\section{AN EXTENSION OF THE EXPANSION Formula}

We begin this section by stating an important property of $T_{3}(k, l)$.

Lemma 3. We have $T_{3}\left(\frac{k}{2}, r \frac{k}{2}\right)=0$, if $k$ is a positive integer and $r \equiv 1(\bmod 4)$.

Proof. The argument is essentially the same as that given in [2, (15)]. 
We will also use this result as part of another expansion of zero. The problem with doing this, however, is that the expansion formula in $\S 3$ only gives expansions for the four products $T_{\varepsilon_{1}} T_{\varepsilon_{2}}$, where $\varepsilon_{1}, \varepsilon_{2} \in\{0,1\}$. Thus, to be able to have an expansion of zero involving $T_{2}$ or $T_{3}$, we must consider whether there is an expansion formula for $T_{\varepsilon_{1}} T_{\varepsilon_{2}}$, when $\varepsilon_{1}, \varepsilon_{2} \in\{0,1,2,3\}$. If one examines the other 12 of the 16 possible products, it becomes clear that there is no expansion in some cases. Since further conditions on the parity of $a, b$, and $m$ (and in some cases even more complicated conditions) are required for other expansion formulas to exist, we will not go into this more general question here. Instead we will confine our discussion to the case we will actually use in the second proof of (9).

Theorem 4. Let $a, b$, and $m \in Z^{+}$, where $a \equiv 2(\bmod 4), b \equiv 1(\bmod 2)$, and $4 \mid \mathrm{m}$. Also, let $\left(k_{1}, l_{1}\right),\left(k_{2}, l_{2}\right) \in\left\{\left(\frac{i}{2}, \frac{j}{2}\right):(i, j) \in Z^{+} \times Z, i \equiv j\right.$ $(\bmod 2)\}$. If the separability condition

$$
k_{1} b=k_{2} a(m-a b)
$$

is satisfied, then

$$
T_{1}\left(k_{1}, l_{1}\right) T_{3}\left(k_{2}, l_{2}\right)=\sum_{r \in R}(-1)^{r(r-1) / 2} x^{k_{2} r^{2}+l_{2} r} T\left(K_{1}, L_{1}(r)\right) T\left(K_{2}, L_{2}(r)\right),
$$

where

$$
\begin{aligned}
K_{1}=k_{1}+k_{2} a^{2}, \quad K_{2} & =k_{2} m(m-a b), \\
L_{1}(r)=l_{1}-l_{2} a-2 k_{2} a r, \quad L_{2}(r) & =\left(2 k_{2} r+l_{2}\right)(m-a b)+l_{1} b,
\end{aligned}
$$

and $R$ is a complete residue system $\bmod m$.

Proof. The proof is substantially the same as the proof in [2, pp. 306-307]. Here, however, the exponent of $(-1)$ in the definition of $T_{3}$ is quadratic in $n$, so the way it transforms when the variables are changed in the proof is more complicated than before. In particular, we have

$$
\begin{aligned}
T_{1}\left(k_{1}, l_{1}\right) T_{3}\left(k_{2}, l_{2}\right) & =\sum_{i}(-1)^{i} x^{k_{1} i^{2}+l_{1} i} \sum_{j}(-1)^{j(j-1) / 2} x^{k_{2} j^{2}+l_{2} j} \\
& =\sum_{i, j}(-1)^{j(j-1) / 2+i} x^{k_{1} i^{2}+l_{1} i+k_{2} j^{2}+l_{2} j}
\end{aligned}
$$

After carrying out the three transformations in the proof (viz. $j=n-a i$, $n=s m+r$, and $i=t+b s$ ) on just the exponent of $(-1)$ (the rest is the same as before), we find that the sign in the final sum is determined by

$$
(-1)^{t+b s+\frac{1}{2}[r+(m-a b) s-a t][r-1+(m-a b) s-a t]} .
$$


Separating this power into its $r, s, t$, and $s t$ factors, we find this is

$$
\begin{aligned}
(-1)^{r(r-1) / 2} & \cdot(-1)^{b s+(m-a b) r s-\frac{1}{2}(m-a b) s+\frac{1}{2}(m-a b)^{2} s^{2}} \\
& \cdot(-1)^{t-a r t+\frac{1}{2} a t+\frac{1}{2} a^{2} t^{2}} \cdot(-1)^{-a(m-a b) s t} .
\end{aligned}
$$

Since $a$ is even, the fourth factor equals 1 . Also, since $b$ is odd and $m-a b \equiv 2$ $(\bmod 4)$, then

$$
(-1)^{b s+(m-a b) r s-\frac{1}{2}(m-a b) s+\frac{1}{2}(m-a b)^{2} s^{2}}=(-1)^{s+0+s+0}=1,
$$

and

$$
(-1)^{t-a r t+\frac{1}{2} a t+\frac{1}{2} a^{2} t^{2}}=(-1)^{t+0+t+0}=1 .
$$

Thus, the sign in the sum indexed by $r$ is given by $(-1)^{r(r-1) / 2}$ while the signs in the sums indexed by $s$ and $t$ are all plus, so the expansion contains only $T_{0}$ 's.

\section{THE SECOND PROOF OF THEOREM 2}

We begin this section by introducing some useful terminology.

Definition. A $T^{2}$ set, or a $T^{2}$ equation, will be called balanced if the $\left(k_{1}, k_{2}\right)$ pair in each of its terms is the same. We will also say that the set or equation is balanced "at $\left(k_{1}, k_{2}\right)$ ".

For example, equation (20) is balanced at $(4,28)$. By analogy, one might also say that equation $(16)$ is a " $Q^{2}$ equation balanced at $(8,56)$ ".

In this section we will prove (9) without first transforming it into a $Q^{2}$ equation. Nonetheless, we will still use the two ideas in the proof of (17), viz. (i) the expansion formula is employed to change a $T^{2}$ set into a balanced $T^{2}$ set; (ii) this balanced set is then shown to be zero by grouping its terms into subsums, each of which is an expansion of zero. Although the ideas in this proof are the same as in the first proof, the new proof is more complicated because of the large number of terms that must be dealt with.

Second Proof of Theorem 2. Our goal here is to verify equation (9) written as

$$
T\left(\frac{21}{2}, \frac{7}{2}\right) T\left(\frac{3}{2}, \frac{3}{2}\right)-x^{2} T\left(\frac{21}{2}, \frac{21}{2}\right) T\left(\frac{3}{2}, \frac{1}{2}\right)-2 T_{1}(21,7) T_{1}(3,1)=0 .
$$

The first step is the same as the beginning of the proof of (15): Expand $T\left(\frac{21}{2}, \frac{7}{2}\right) T\left(\frac{3}{2}, \frac{3}{2}\right)$ and $x^{2} T\left(\frac{21}{2}, \frac{21}{2}\right) T\left(\frac{3}{2}, \frac{1}{2}\right)$ with $[1,1,8]$ and cancel terms in $T\left(\frac{21}{2}, \frac{7}{2}\right) T\left(\frac{3}{2}, \frac{3}{2}\right)-x^{2} T\left(\frac{21}{2}, \frac{21}{2}\right) T\left(\frac{3}{2}, \frac{1}{2}\right)$ to obtain (15). Next, expand all but the first and fifth terms on the right side of (15) with $k_{1}=84, k_{2}=12$ and $[1,1,8]$. Expand the first and fifth terms in $(15)$, i.e., $T(84,14) T(12,2)$ and $x^{16} T(84,70) T(12,10)$, with $k_{1}=84, k_{2}=12$ and $[7,1,8]$. (For convenience, we write the factor $T(96, *)$ first in the $T \cdot T$ pairs in the second expansion.) We now write $A_{i}(x)$ and $B_{i}(x), 1 \leq i \leq 48$, for the surviving terms in the expansions of $T\left(\frac{21}{2}, \frac{7}{2}\right) T\left(\frac{3}{2}, \frac{3}{2}\right)$ and $-x^{2} T\left(\frac{21}{2}, \frac{21}{2}\right) T\left(\frac{3}{2}, \frac{1}{2}\right)$, respectively. 
Here, each of the $48 A_{i}$-terms (all are positive) and $48 B_{i}$-terms (all are negative) have the form $x^{\alpha_{i}} T\left(96, L_{1 i}\right) T\left(672, L_{2 i}\right)$. (These are listed in Tables 1 and 2 respectively.) Expanding the third term, $-2 T_{1}(21,7) T_{1}(3,1)$, in (23) with [5, 5, 32] gives the $G_{i}(x)$ terms of the form $\pm x^{\alpha_{i}} T\left(96, L_{1 i}\right) T\left(672, L_{2 i}\right)$ listed in Table 3. (Each of these appears twice because of the coefficient 2.)

At this point we have $160(=48+48+2 \cdot 32)$ terms in the expansion, viz.

$$
\begin{gathered}
T\left(\frac{21}{2}, \frac{7}{2}\right) T\left(\frac{3}{2}, \frac{3}{2}\right)-x^{2} T\left(\frac{21}{2}, \frac{21}{2}\right) T\left(\frac{3}{2}, \frac{1}{2}\right)-2 T_{1}(21,7) T_{1}(3,1) \\
\quad=\sum_{i=1}^{48} A_{i}(x)+\sum_{i=1}^{48} B_{i}(x)+2 \sum_{i=1}^{32} G_{i}(x) .
\end{gathered}
$$

Thirty-two of these positive and negative $G_{i}$ 's cancel with the (positive) $A_{i}$ 's and the (negative) $B_{i}$ 's. (The terms that cancel are indicated by ' $\mathrm{C}$ ' in Tables $1-3$. In Table 3, those $G$ 's numbered $G_{1}, \ldots, G_{16}$ cancel once and those numbered $G_{17}, \ldots, G_{24}$ cancel twice.) This leaves 96 uncanceled terms in the three tables. We next group these terms into three classes labeled " $D$ ", "E", and " $F "$ " The sum of the terms in each of these three classes is zero, because each sum is an expansion of zero. In particular, the expansions of $-2 x^{2} T_{1}(42,14) T_{1}(6,6)$ with $[3,3,16]$, as well as $-T_{1}(42,7) T_{3}\left(\frac{3}{2}, \frac{3}{2}\right)$ and $x^{7} T_{1}(42,35) T_{3}\left(\frac{3}{2}, \frac{3}{2}\right)$ with $[6,3,32]$, exactly give the groups $\mathrm{D}, \mathrm{E}$ and $\mathrm{F}$ respectively, i.e.,

$$
\begin{aligned}
-2 x^{2} T_{1}(42,14) T_{3}(6,6) & =2 \sum_{i=1}^{16} D_{i}(x), \\
-T_{1}(42,7) T_{3}\left(\frac{3}{2}, \frac{3}{2}\right) & =\sum_{i=1}^{32} E_{i}(x),
\end{aligned}
$$

and

$$
x^{7} T_{1}(42,35) T_{3}\left(\frac{3}{2}, \frac{3}{2}\right)=\sum_{i=1}^{32} F_{i}(x) .
$$

(Note that each of the terms denoted by $\mathrm{D}$ appears twice in Tables 2 and 3.) Thus, we find that

$$
\begin{aligned}
T\left(\frac{21}{2},\right. & \left.\frac{7}{2}\right) T\left(\frac{3}{2}, \frac{3}{2}\right)-x^{2} T\left(\frac{21}{2}, \frac{21}{2}\right) T\left(\frac{3}{2}, \frac{1}{2}\right)-2 T_{1}(21,7) T_{1}(3,1) \\
= & 2 \sum_{i=1}^{16} D_{i}(x)+\sum_{i=1}^{32} E_{i}(x)+\sum_{i=1}^{32} F_{i}(x) \\
= & -2 x^{2} T_{1}(42,14) T_{1}(6,6)-T_{1}(42,7) T_{3}\left(\frac{3}{2}, \frac{3}{2}\right) \\
& +x^{7} T_{1}(42,35) T_{3}\left(\frac{3}{2}, \frac{3}{2}\right)=0
\end{aligned}
$$

since each term in the final trinomial is zero by Lemmas 2 and 3 with $r=1$. This establishes (9). 
TABLE $1 . \quad A_{i}(x)=x^{\alpha_{i}} T\left(96, L_{1 i}\right) T\left(672, L_{2 i}\right)$

All the terms in this table are positive

$T(84,14) T(12,2),[7,1,8]$

\begin{tabular}{c|crrr}
$i$ & type & $\alpha_{i}$ & $L_{1 i}$ & $L_{2 i}$ \\
\hline 1. & $\mathrm{E}$ & 0 & 16 & 0
\end{tabular}

2. $\mathrm{F} \quad 10 \quad 8 \quad 168$

3. $\mathrm{F} \quad 14 \quad 40 \quad 168$

4. $F \quad \begin{array}{llll} & \mathrm{F} & 32 & 336\end{array}$

5. $\mathrm{F} \quad 52 \quad 64 \quad 336$

6. F $\quad 102 \quad 56 \quad 504$

7. $\mathrm{F} \quad 114 \quad 88 \quad 504$

8. $\mathrm{E} \quad 184 \quad 80 \quad 672$

$x^{3} T(84,35) T(12,1),[1,1,8]$

\begin{tabular}{r|crrr}
$i$ & type & $\alpha_{i}$ & $L_{1 i}$ & $L_{2 i}$ \\
\hline 17. & $\mathrm{E}$ & 3 & 34 & 42
\end{tabular}

18. $\mathrm{C} \quad 14 \quad 58 \quad 126$

19. C $16 \quad 10 \quad 210$

20. E $49 \begin{array}{lll}49 & 294\end{array}$

21. $\begin{array}{llll}\text { E } & 53 & 14 & 378\end{array}$

22. $\mathrm{C} \quad 98 \quad 86 \quad 462$

23. $\mathrm{C} \quad 114 \quad 38 \quad 546$

24. $\mathrm{E} \quad 157 \quad 62 \quad 630$

$x^{16} T(84,70) T(12,10),[7,1,8]$

\begin{tabular}{r|crrr}
$i$ & type & $\alpha_{i}$ & $L_{1 i}$ & $L_{2 i}$ \\
\hline 33. & $\mathrm{~F}$ & 16 & 80 & 0
\end{tabular}

$\begin{array}{lllll}34 . & \mathrm{E} & 18 & 56 & 168\end{array}$

$\begin{array}{lllll}35 . & \mathrm{E} & 30 & 88 & 168\end{array}$

36. $\mathrm{E} \quad 44 \quad 32 \quad 336$

37. $\mathrm{E} \quad 52 \quad 64 \quad 336$

38. $\mathrm{E} \quad 94 \quad 8 \quad 504$

39. $\mathrm{E} \quad 98 \quad 40 \quad 504$

40. $\mathrm{F} \quad 168 \quad 16 \quad 672$
$T(84,7) T(12,5),[1,1,8]$

\begin{tabular}{c|crrr}
$i$ & type & $\alpha_{i}$ & $L_{1 i}$ & $L_{2 i}$ \\
\hline 9. & $\mathrm{C}$ & 0 & 2 & 42
\end{tabular}

10. $\mathrm{F} \quad 7 \quad 26 \quad 126$

11. F F $17 \quad 22 \quad 210$

12. $\mathrm{C} \quad 38 \quad 50 \quad 294$

13. $\begin{array}{llll}\mathrm{C} & 58 & 46 & 378\end{array}$

14. $\begin{array}{llll}\mathrm{F} & 93 & 74 & 462\end{array}$

15. $\begin{array}{llll}\text { F } & 123 & 70 & 546\end{array}$

16. $\begin{array}{llll}\text { C } & 170 & 94 & 630\end{array}$

$x^{9} T(84,49) T(12,11),[1,1,8]$

\begin{tabular}{r|crrr}
$i$ & type & $\alpha_{i}$ & $L_{1 i}$ & $L_{2 i}$ \\
\hline 25. & $\mathrm{E}$ & 9 & 38 & 126
\end{tabular}

26. C C $10 \quad 62 \quad 42$

27. $\begin{array}{llll}C & 32 & 14 & 294\end{array}$

28. $\begin{array}{llll}\text { E } & 35 & 86 & 210\end{array}$

29. $\mathrm{C} \quad 70 \quad 82 \quad 378$

30. E $79 \quad 10 \quad 462$

31. $\quad$ E $119 \quad 58 \quad 546$

32. $\mathrm{C} \quad 150 \quad 34 \quad 630$

$x^{18} T(84,77) T(12,7),[1,1,8]$

\begin{tabular}{r|cccc}
$i$ & type & $\alpha_{i}$ & $L_{1 i}$ & $L_{2 i}$ \\
\hline 41. & $\mathrm{C}$ & 18 & 70 & 126
\end{tabular}

42. $\begin{array}{llll}F & 23 & 94 & 42\end{array}$

43. $\mathrm{C} \quad 30 \quad 74 \quad 210$

44. $F \quad \begin{array}{llll} & 37 & 46 & 294\end{array}$

45. $F \quad \begin{array}{llll}59 & 50 & 378\end{array}$

46. $\begin{array}{llll}\text { C } & 80 & 22 & 462\end{array}$

47. $\begin{array}{llll}\text { C } & 112 & 26 & 546\end{array}$

48. $\begin{array}{llll}\text { F } & 147 & 2 & 630\end{array}$ 
TABLE 2. $\quad B_{i}(x)=x^{\alpha_{i}} T\left(96, L_{1 i}\right) T\left(672, L_{2 i}\right)$

All the terms in this table are negative

$x^{2} T(84,14) T(12,10),[1,1,8]$

\begin{tabular}{r|crrr}
$i$ & type & $\alpha_{i}$ & $L_{1 i}$ & $L_{2 i}$ \\
\hline 1. & $\mathrm{D}$ & 2 & 4 & 84 \\
2. & $\mathrm{C}$ & 4 & 28 & 84 \\
3. & $\mathrm{C}$ & 24 & 20 & 252 \\
4. & $\mathrm{D}$ & 30 & 52 & 252 \\
5. & $\mathrm{D}$ & 70 & 44 & 420 \\
6. & $\mathrm{C}$ & 80 & 76 & 420 \\
7. & $\mathrm{C}$ & 140 & 68 & 588 \\
8. & $\mathrm{D}$ & 150 & 92 & 588
\end{tabular}

$x^{4} T(84,35) T(12,7),[1,1,8]$

\begin{tabular}{r|rrrr}
$i$ & type & $\alpha_{i}$ & $L_{1 i}$ & $L_{2 i}$ \\
\hline 17. & $\mathrm{C}$ & 4 & 28 & 84 \\
18. & $\mathrm{E}$ & 9 & 52 & 84 \\
19. & $\mathrm{F}$ & 23 & 4 & 252 \\
20. & $\mathrm{D}$ & 38 & 76 & 252 \\
21. & $\mathrm{D}$ & 66 & 20 & 420 \\
22. & $\mathrm{F}$ & 87 & 92 & 420 \\
23. & $\mathrm{E}$ & 133 & 44 & 588 \\
24. & $\mathrm{C}$ & 140 & 68 & 588
\end{tabular}

$x^{14} T(84,70) T(12,2),[1,1,8]$

\begin{tabular}{r|crrr}
$i$ & type & $\alpha_{i}$ & $L_{1 i}$ & $L_{2 i}$ \\
\hline 33. & $\mathrm{D}$ & 14 & 68 & 84
\end{tabular}

34. $\mathrm{C} \quad 24 \quad 92 \quad 84$

35. $\mathrm{C} \quad 28 \quad 44 \quad 252$

36. $\mathrm{D} \quad 38 \quad 76 \quad 252$

37. D $66 \quad 20 \quad 420$

38. $\mathrm{C} \quad 72 \quad 52 \quad 420$

39. $\mathrm{C} \quad 128 \quad 4 \quad 588$

40. $\mathrm{D} \quad 130 \quad 28 \quad 588$ $x^{2} T(84,7) T(12,11),[1,1,8]$

\begin{tabular}{r|crrr}
$i$ & type & $\alpha_{i}$ & $L_{1 i}$ & $L_{2 i}$ \\
\hline 9. & $\mathrm{D}$ & 2 & 4 & 84 \\
10. & $\mathrm{E}$ & 3 & 20 & 84 \\
11. & $\mathrm{F}$ & 25 & 28 & 252 \\
12. & $\mathrm{C}$ & 28 & 44 & 252 \\
13. & $\mathrm{C}$ & 72 & 52 & 420 \\
14. & $\mathrm{F}$ & 77 & 68 & 420 \\
15. & $\mathrm{E}$ & 143 & 76 & 588 \\
16. & $\mathrm{D}$ & 150 & 92 & 588
\end{tabular}

$x^{7} T(84,49) T(12,5),[1,1,8]$

\begin{tabular}{r|rrrr}
$i$ & type & $\alpha_{i}$ & $L_{1 i}$ & $L_{2 i}$ \\
\hline 25. & $\mathrm{~F}$ & 7 & 44 & 84 \\
26. & $\mathrm{D}$ & 14 & 68 & 84 \\
27. & $\mathrm{C}$ & 24 & 20 & 252 \\
28. & $\mathrm{E}$ & 45 & 92 & 252 \\
29. & $\mathrm{C}$ & 80 & 76 & 420 \\
30. & $\mathrm{E}$ & 65 & 4 & 420 \\
31. & $\mathrm{D}$ & 130 & 28 & 588 \\
32. & $\mathrm{~F}$ & 135 & 52 & 588
\end{tabular}

$x^{17} T(84,77) T(12,1),[1,1,8]$

\begin{tabular}{r|rrrr}
$i$ & type & $\alpha_{i}$ & $L_{1 i}$ & $L_{2 i}$ \\
\hline 41. & $\mathrm{~F}$ & 17 & 76 & 84 \\
42. & $\mathrm{C}$ & 24 & 92 & 84 \\
43. & $\mathrm{D}$ & 30 & 52 & 252 \\
44. & $\mathrm{E}$ & 35 & 68 & 252 \\
45. & $\mathrm{E}$ & 67 & 28 & 420 \\
46. & $\mathrm{D}$ & 70 & 44 & 420 \\
47. & $\mathrm{C}$ & 128 & 4 & 588 \\
48. & $\mathrm{~F}$ & 129 & 20 & 588
\end{tabular}


TABLE 3. $G_{i}(x)= \pm x^{\alpha_{i}} T\left(96, L_{1 i}\right) T\left(672, L_{2 i}\right)$

$$
-2 T_{1}(21,7) T_{1}(3,1), \quad[5,5,32]
$$

Each term occurs twice

Negative Terms

\begin{tabular}{r|rrrr}
$i$ & type & $\alpha_{i}$ & $L_{1 i}$ & $L_{2 i}$ \\
\hline 1. & $\mathrm{CE}$ & 0 & 2 & 42 \\
2. & $\mathrm{CF}$ & 10 & 62 & 42 \\
3. & $\mathrm{CF}$ & 14 & 58 & 126 \\
4. & $\mathrm{CF}$ & 16 & 10 & 210 \\
5. & $\mathrm{CE}$ & 18 & 70 & 126 \\
6. & $\mathrm{CE}$ & 30 & 74 & 210 \\
7. & $\mathrm{CF}$ & 32 & 14 & 294 \\
8. & $\mathrm{CE}$ & 38 & 50 & 294 \\
9. & $\mathrm{CE}$ & 58 & 46 & 378 \\
10. & $\mathrm{CF}$ & 70 & 82 & 378 \\
11. & $\mathrm{CE}$ & 80 & 22 & 462 \\
12. & $\mathrm{CF}$ & 98 & 86 & 462 \\
13. & $\mathrm{CE}$ & 112 & 26 & 546 \\
14. & $\mathrm{CF}$ & 114 & 38 & 546 \\
15. & $\mathrm{CF}$ & 150 & 34 & 630 \\
16. & $\mathrm{CE}$ & 170 & 94 & 630
\end{tabular}

Positive Terms

\begin{tabular}{r|rrrr}
$i$ & type & $\alpha_{i}$ & $L_{1 i}$ & $L_{2 i}$ \\
\hline 17. & CC & 4 & 28 & 84 \\
18. & CC & 24 & 20 & 252 \\
19. & CC & 24 & 92 & 84 \\
20. & CC & 28 & 44 & 252 \\
21. & CC & 72 & 52 & 420 \\
22. & CC & 80 & 76 & 420 \\
23. & CC & 140 & 68 & 588 \\
24. & CC & 128 & 4 & 588 \\
25. & DD & 2 & 32 & 0 \\
26. & DD & 14 & 40 & 168 \\
27. & DD & 30 & 88 & 168 \\
28. & DD & 42 & 16 & 336 \\
29. & DD & 58 & 80 & 336 \\
30. & DD & 94 & 8 & 504 \\
31. & DD & 102 & 56 & 504 \\
32. & DD & 178 & 64 & 672
\end{tabular}

\section{COMMENTARY ON THE SECOND PROOF}

The problem of putting together the second proof was solved by first finding what expansions could be used on the left of $(23)$ and then discovering how the resulting terms could be grouped into subsums that were expansions of zero. To help in analyzing this problem, we devised two algorithms, Forward and Backward.

The variables $k_{1}, k_{2}, K_{1}$, and $K_{2}$ used in both these algorithms are positive multiples of $\frac{1}{2}$. When $x$ and $y$ are rational, the notation $x \mid y$ means $\frac{y}{x}$ is an integer.

Algorithm Forward. Given $k_{1}, k_{2}$, and bound $M$. Find all values of $K_{1}$, $K_{2}, a, b$, and $m \leq M$ that satisfy (11) and the first two equations in (12).

for $m=2$ to $M$ do

$$
\begin{aligned}
& \text { for } a=1 \text { to } m-1 \text { do } \\
& K_{1}=k_{1}+k_{2} a^{2} \\
& \text { if } K_{1} \mid a k_{2} m \text { then } \\
& b=\frac{a k_{2} m}{K_{1}}, \quad K_{2}=k_{2} m(m-a b) \\
& \text { output } K_{1}, K_{2}, a, b, m
\end{aligned}
$$


Proof. To verify the algorithm, we need only show that (11) is satisfied:

$$
b=\frac{a k_{2} m}{K_{1}} \Longrightarrow b\left(k_{1}+k_{2} a^{2}\right)=a k_{2} m \Longrightarrow k_{1} b=k_{2} a(m-a b) \text {. }
$$

The Backward program is somewhat more elaborate, but needs no bound, because, given $K_{1}$ and $K_{2}$, the formulas in (12) clearly show that only a finite number of positive integers can satisfy them.

Algorithm Backward. Given $K_{1}, K_{2}$. Find all possible values of $k_{1}, k_{2}, a$, $b, m$ that satisfy (11) and the first two equations in (12).

for $a$ such that $1 \leq a^{2}<K_{1}$ do

for $k_{2}$ such that $\frac{1}{2} \leq a^{2} k_{2}<K_{1}$ and $k_{2} \mid K_{2}^{*}$ do

$$
\begin{aligned}
& k_{1}=K_{1}-k_{2} a^{2} \\
& \text { if } \frac{k_{2} K_{2}}{k_{1} K_{1}}=s^{2} \text {, a perfect square, then } \\
& b=a s, m=\frac{a K_{2}}{b k_{1}} \\
& \quad \text { output } k_{1}, k_{2}, a, b, m
\end{aligned}
$$

Proof. Since $K_{1}=k_{1}+k_{2} a^{2}$ and $k_{1}, k_{2}>0$, the right-hand side in this equation is $>0$, so there are at most a finite number of values for $k_{1}, k_{2}$ and $a$ for which the equation holds. We must show that the numbers $k_{1}, k_{2}, a, b$, and $m$ satisfy the required conditions. There is nothing to prove for $k_{1}$, considering how it was obtained. To show (11): $b^{2}=\frac{a^{2} k_{2} K_{2}}{k_{1} K_{1}} \Longrightarrow b K_{1}=\frac{a^{2} k_{2} K_{2}}{b k_{1}}=a k_{2} m$. Thus,

$$
b\left(k_{1}+k_{2} a^{2}\right)=a k_{2} m \Longrightarrow k_{1} b=k_{2} a(m-a b) .
$$

The second equation in (12) now follows, since

$$
b k_{1} m=a k_{2} m(m-a b) \Longrightarrow k_{2} m(m-a b)=\frac{b k_{1} m}{a}=K_{2} \text {. }
$$

Used together, these programs allow the tracing of composition "paths"starting with $\left(k_{1}, k_{2}\right)$ and ending at $\left(K_{1}, K_{2}\right)$-through all possible intermediate pairs, which are discovered by using Backward on $\left(K_{1}, K_{2}\right)$. Our first problem is to turn the $T^{2}$ equation (23) into a balanced equation. The question is: At what $K$-pair should the equation be balanced? In the first proof we were able to expand just the right-hand side of (17) to balance the equation at $(4,28)$. This is the first possibility to consider here: Starting with $k_{1}=\frac{21}{2}$, $k_{2}=\frac{3}{2}\left(\right.$ or $\left.k_{1}=\frac{3}{2}, k_{2}=\frac{21}{2}\right)$, can we expand to $K_{1}=21, K_{2}=3$, the $K$-pair of the third term? Using (12), we would have to have either

$$
21=\frac{21}{2}+\frac{3}{2} a^{2} \Longrightarrow a^{2}=7 \Longrightarrow a \notin Z^{+}
$$

*For example, if $K_{2}=15$, then the positive values of $k_{2}$ such that $k_{2} \mid K_{2}$ are 1, 3, 5, 15, $\frac{1}{2}, \frac{3}{2}, \frac{5}{2}, \frac{15}{2}$. 
or

$$
21=\frac{3}{2}+\frac{21}{2} a^{2} \Longrightarrow a^{2}=\frac{13}{7} \Longrightarrow a \notin Z^{+} .
$$

Thus, an expansion of just the first two terms of (23) will not work. We therefore conclude that all the terms will have to be expanded to terms with a common $K$-pair if equation (23) is to be expanded to a balanced equation. The next question is: What are the possible common $K$-pairs? To determine this, we used Forward to find all expansions of $\left(\frac{21}{2}, \frac{3}{2}\right)$ with their parameters for $2 \leq m \leq 64$ (the complete set of values are listed in Table 4A only for $2 \leq m \leq 32$ and $m=64)$ and of $(21,3)$ with their parameters for $2 \leq m \leq 32$ (these are given in Table 4B). These tables show very well just how restrictive condition (11) is. Actually, it would be important (and remarkable) if there were always a balanced equation to which one could expand from a $T^{2}$ equation in which all the terms cancel directly, making any further proof unnecessary (cf. [2, (37)]).

TABLE 4A. Forward

\begin{tabular}{rr|rrr}
\multicolumn{1}{l}{$k_{1}=\frac{21}{2}, \quad k_{2}=\frac{3}{2}}$, & \multicolumn{3}{c}{$2 \leq m \leq 64$} \\
$K_{1}$ & $K_{2}$ & $a$ & $b$ & $m$ \\
\hline$\rightarrow 12$ & 84 & 1 & 1 & 8 \\
$\rightarrow 84$ & 12 & 7 & 1 & 8 \\
$33 / 2$ & $231 / 2$ & 2 & 2 & 11 \\
12 & 336 & 1 & 2 & 16 \\
$* 24$ & 168 & 3 & 3 & 16 \\
84 & 48 & 7 & 2 & 16 \\
$33 / 2$ & 462 & 2 & 4 & 22 \\
$69 / 2$ & $483 / 2$ & 4 & 4 & 23 \\
12 & 756 & 1 & 3 & 24 \\
84 & 108 & 7 & 3 & 24 \\
$609 / 2$ & $87 / 2$ & 14 & 2 & 29 \\
12 & 1344 & 1 & 4 & 32 \\
$* 24$ & 672 & 3 & 6 & 32 \\
$* 48$ & 336 & 5 & 5 & 32 \\
84 & 192 & 7 & 4 & 32 \\
$\vdots$ & $\vdots$ & $\vdots$ & $\vdots$ & $\vdots$ \\
$* 24$ & 2688 & 3 & 12 & 64 \\
$* 48$ & 1344 & 5 & 10 & 64 \\
84 & 768 & 7 & 8 & 64 \\
$* 672$ & 96 & 21 & 3 & 64
\end{tabular}

TABLE 4B. Forward

$$
k_{1}=21, \quad k_{2}=3, \quad 2 \leq m \leq 32
$$

\begin{tabular}{rr|rrr}
$K_{1}$ & $K_{2}$ & $a$ & $b$ & $m$ \\
\hline$* 24$ & 168 & 1 & 1 & 8 \\
$* 168$ & 24 & 7 & 1 & 8
\end{tabular}

\begin{tabular}{ll|lll}
33 & 231 & 2 & 2 & 11
\end{tabular}

\begin{tabular}{ll|lll}
$* 24$ & 672 & 1 & 2 & 16
\end{tabular}

\begin{tabular}{ll|lll}
$* 48$ & 336 & 3 & 3 & 16
\end{tabular}

\begin{tabular}{ll|lll}
168 & 96 & 7 & 2 & 16
\end{tabular}

\begin{tabular}{ll|lll}
33 & 924 & 2 & 4 & 22
\end{tabular}

\begin{tabular}{ll|lll}
69 & 483 & 4 & 4 & 23
\end{tabular}

\begin{tabular}{ll|lll}
24 & 1512 & 1 & 3 & 24
\end{tabular}

\begin{tabular}{ll|lll}
168 & 216 & 7 & 3 & 24
\end{tabular}

\begin{tabular}{ll|lll}
609 & 87 & 14 & 2 & 29
\end{tabular}

*24 $2688 \quad 1 \quad 4 \quad 32$

\begin{tabular}{ll|lll}
$* 48$ & 1344 & 3 & 6 & 32
\end{tabular}

\begin{tabular}{ll|lll}
$* 96$ & 672 & 5 & 5 & 32
\end{tabular}

\begin{tabular}{ll|lll}
168 & 384 & 7 & 4 & 32
\end{tabular}


TABLE 5. Backward

\begin{tabular}{rr|rrr}
\multicolumn{3}{c}{$K_{1}=672$} & \multicolumn{3}{c}{$K_{2}=96$} \\
$k_{1}$ & $k_{2}$ & $a$ & $b$ & $m$ \\
\hline$\rightarrow 84$ & 12 & 1 & 1 & 8 \\
$\rightarrow 12$ & 84 & 1 & 7 & 8 \\
84 & 3 & 2 & 1 & 16 \\
12 & 21 & 2 & 7 & 16 \\
42 & 6 & 3 & 3 & 16 \\
$3 / 2$ & $21 / 2$ & 3 & 21 & 64 \\
21 & 3 & 5 & 5 & 32 \\
42 & $3 / 2$ & 6 & 3 & 32
\end{tabular}

$$
K_{1}=96 \quad K_{2}=672
$$

\begin{tabular}{rr|rrr}
$k_{1}$ & $k_{2}$ & $a$ & $b$ & $m$ \\
\hline$\rightarrow 84$ & 12 & 7 & 1 & 8 \\
84 & 3 & 14 & 1 & 16 \\
$21 / 2$ & $3 / 2$ & 21 & 3 & 64
\end{tabular}

An examination of the $k$-pairs that are common to Tables 4A and 4B (indicated by an asterisk) shows that the smallest such pair is $(24,168)$ (or possibly $(168$, $24)$ ), which occurs with $[3,3,16]$ in Table $4 A$ and with both $[1,1,8]$ and $[7,1,8]$ in Table 4B. Thus, there are two possible expansions to examine. To establish either of the resulting equations by means of expansions of zero, we must use Backward to find all the $k$-pair "ancestors" of $K_{1}=24, K_{2}=168$ (or $K_{1}=$ $\left.168, K_{2}=24\right)$ to use in this expansion. For $(24,168)$, there are the three ancestors:

$$
\begin{gathered}
k_{1}=21, k_{2}=3 \text { with }[1,1,8], \quad k_{1}=3, k_{2}=21 \text { with }[1,7,8], \\
\quad \text { and } k_{1}=\frac{21}{2}, k_{2}=\frac{3}{2} \text { with }[3,3,16] .
\end{gathered}
$$

For $(168,24)$ there is only $k_{1}=21, k_{2}=3$ with $[7,1,8]$. After examining all the possible expansions of zero, we find that none of them gives a proof of (23).

Thus, we must try another, larger $K$-pair from Tables 4A and 4B. This time we chose $K_{1}=96, K_{2}=672$ because Backwards gives a large collection of ancestors from which we can try to construct a proof. Although $m$ has the large value 64 for this $K$-pair in Table 4A, there is a second way to expand the first two terms, viz. by the composition of two expansions: the first from $\left(\frac{21}{2}, \frac{3}{2}\right)$ to $(12,84)$ and the second from $(12,84)$ to $(672,96)$. (These entries are indicated by arrows in Tables $4 \mathrm{~A}$ and 5.) Even though it would seem that 64 terms would also be produced by this composition, it happens that some terms cancel after the first expansion, so there are actually only 96 terms that must be dealt with. We expand the third term in $(23)$ to $(96,672)$ using the parameters $[5,5,32]$ in Table 4B. When all the terms are combined, the 96 remaining terms must then be examined to see if they can be grouped into expansions of zero.

It is sometimes the case in this examination that a certain subsum of terms stands out as a candidate for such a set, as in the present case where the doubled 
(D) terms form a noticeable set. From Table 3 the sum of the D terms is

$$
\begin{aligned}
D^{+} \stackrel{\text { def }}{=} & (2,32,0)+(14,40,168)+(30,88,168) \\
& +(42,16,336)+(58,80,336)+(94,8,504) \\
& +(102,56,504)+(178,64,672)
\end{aligned}
$$

and the sum in Table 2 is

$$
\begin{aligned}
D^{-} \stackrel{\text { def }}{=} & (2,4,84)+(30,52,252)+(70,44,420) \\
& +(150,92,588)+(38,76,252)+(66,20,420) \\
& +(14,68,84)+(130,28,588) .
\end{aligned}
$$

(Here the triple $\left(\alpha, L_{1}, L_{2}\right)$ is an abbreviated notation for the term $x^{\alpha} T\left(96, L_{1}\right) T\left(672, L_{2}\right)$.) Since the $D^{-}$terms are negative in Table 2, the question is whether $D^{+}-D^{-}$is an expansion of zero, say based on $T_{1}\left(T_{3}\right.$ is the other possibility). If so, we might have

$$
D^{+}-D^{-}= \pm x^{2} T_{1}\left(k_{1}, l_{1}\right) T_{1}\left(k_{2}, k_{2}\right)=0,
$$

with the sign to be determined later. If this were the case, it would then follow that $D^{+}+D^{-}=x^{2} T_{0}\left(k_{1}, l_{1}\right) T_{0}\left(k_{2}, k_{2}\right)$, i.e., the sum with the same terms, but now with all plus signs. Adding these equations gives

$$
x^{-2} D^{+}=\frac{1}{2} T_{0}\left(k_{1}, l_{1}\right) T_{0}\left(k_{2}, k_{2}\right)=T_{0}\left(k_{1}, l_{1}\right) T_{0}\left(4 k_{2}, 2 k_{2}\right),
$$

using (4), i.e., the assumption that $D^{+}-D^{-}$vanishes because it is an expansion of zero of this kind implies that the power series for $x^{-2} D^{+}$must factor into a $T_{0} \cdot T_{0}$ product. The factorization of this power series up to degree 1000 by the greedy algorithm (cf. [2, p. 311]) indicates that $x^{-2} D^{+}$is undoubtedly equal to $(0, \pm 48, \pm 96, \pm 144,168)_{336}(0)_{168}[ \pm 12, \pm 28, \pm 36, \pm 56, \pm 60]_{168}$, which is $(0)_{84}[ \pm 28]_{84}(0)_{48}[ \pm 12]_{48}=T_{0}(42,6) T_{0}(24,12)$. Thus, we conclude that $k_{1}=42, l_{1}=14$, and $k_{2}=6$. Expanding the $T_{1} \cdot T_{1}$ product with $[3,3,16]$ (these parameters being computed from (11) and (12)) determines that the sign in (27) is negative: $D^{+}-D^{-}=-x^{2} T_{1}(42,14) T_{1}(6,6)$.

Of course, if the form for the expansion of zero we tried in (27) were not correct, this would become apparent when $D^{+}$was factored; for not only would the expected form on the right not occur, but rather, the factorization would undoubtedly be the product of binomials in which the sequence of exponents increased, apparently without bound.

In the general case, where no particular subsum is a candidate for an expansion of zero, we can first find all the ancestors of $\left(K_{1}, K_{2}\right)$ and then examine all the possible expansions of zero to see if the full $T^{2}$ set can be written as a combination of some of them. This is how the last two expansions of zero in the proof were found-the first being the sum of the doubles.

The irregularities in the proof, such as using two different but related expansions in the second expansion of the composition, and using $T_{3}$ in an expansion of zero, were found to be necessary to match the signs of the terms so a proof could be made by this method. 


\section{BIBLIOGRAPHY}

1. R. Blecksmith, J. Brillhart, and I. Gerst, Parity results for certain partition functions and identities similar to theta function identities, Math. Comp. 48 (1987), 29-38.

2. __ Some infinite product identities, Math. Comp. 51 (1988), 301-314.

3. _ On the $\bmod 2$ reciprocation of infinite modular-part products and the parity of certain partition functions, Math. Comp. 54 (1990), 345-376.

4. __ On a certain $(\bmod 2)$ identity, Abstracts Amer. Math. Soc. 11 (1990), 145.

Department of Mathematical Sciences, Northern Illinois University, DeKalb, IlliNOIS 60115

Department of Mathematics, University of Arizona, Tucson, Arizona 85721

Department of Applied Mathematics and Statistics, SUNY at Stony Brook, Stony BROOK, NEW YORK 11794 